\title{
Beiträge zur Erforschung der germanischen Lehnwörter im Ostseefinnischen
}

\section{1. lukko}

Das fi. Wort lukko hat neben der allgemeinen Bedeutung 'Schloss' auch die Bedeutung 'Schlucht zwischen Landrücken'. Das NS (= Nykysuomen sanakirja, "Wörterbuch der finnischen Gegenwartssprache"), das auch die letztgenannte Bedeutung zur Standardsprache rechnet, behandelt diese beiden Wörter unter einem Lemma. Als eine mögliche Begründung könnte man annehmen, dass die 'Schlucht zwischen Landrücken' ein 'Schloss' im übertragenen Sinne wäre: in einer solchen Schlucht kann man ja sich gewissermassen 'eingeschlossen' fühlen.

Lukko 'Schloss' gilt von alters her als ein LW (= Lehnwort) aus den germ. Sprachen. Lediglich was die Datierung der Entlehnung betrifft, gehen die Meinungen auseinander: 1t. Karsten (1943 - 44 299) wurde lukko aus dem Altschwedischen entlehnt, das SKES (= Suomen kielen etymologinen sanakirja, "Etymologisches Wörterbuch der finnischen Sprache", II 308) hält es für ein "skandinavisches" LW, während Raun (1958 32), Hakulinen (1960 55 und 1979 360), Vries (1962 364 - 365), Raun - Saareste (1965 44) und K. Vilkuna (KLM XXI 1977 275) es zu den alten germ. LW zählen. Aus dem Germanischen nennt das SKES vergleichend an. lok, loka 'Riegel, Schloss', norw. loka 'Riegel, Querholz' und schwed. dial. luka 'Riegel (aus Holz)'.

Jorma Koivulehto äussert in seiner Dissertation (1971 22 - 23) nebenbei die Vermutung, dass lukko 'Schlucht' (nach Koivulehto 'eine von allen Seiten umschlossene tiefe Einsenkung im Osgelände, oft mit feuchtem Grund') ein germ. LW sei. Dieses lukko wäre nach Koivulehto aus der germ. Form *luko- >*luka- entlehnt, die 
z.B. in dt. Loch und an. lok ('Schluss, Ende', Vries 1962 364) belegt ist. In SKES (II 307 - 308; diese Lieferung erschien 1958) wird lukko 'Schlucht' unter lukka behandelt; dieser Wortartikel lautet in extenso wie folgt: "lukka '(von unten hohler) überstehender Rand einer Sandgrube oder eines Grabes' (mies oli lukan alla'der Mann war unter lukka', hauta kaivettiin lukalle 'man grub den Graben mit lukka', kaivettiin lapsen arkulle sija lukan alle 'man grub einen Platz für den Sarg des Kindes unter lukka'), lukko 'Furche, Nut, Grube, tiefes Tal', luko 'kleine Schlucht, Vertiefung' $=$ ? tscher. là k $k$, lakẹ 'Grube, Vertiefung, Schlucht' / ? ung. dial. lok 'enger Raum zwischen zwei Bergen, Ebene, Tal'.' Das SKES führt also aus zwei fiu. Sprachen unsichere Entsprechungen an, die hinsichtlich der Bedeutung gerade zu lukko 'Schlucht' passen würden. Da der fiu. Ursprung des Wortes lukko als sehr fraglich zu gelten hat (hinsichtlich des ung. Wortes vgl. auch MNyTESz II 784 und Kniezsa 1974 879), ist es berechtigt, die Herkunft von lukko nur vom Finnischen ausgehend zu betrachten. Hierbei ist es allerdings angebracht, all die Wörter, die im SKES unter lukka behandelt werden, mit in die Betrachtung einzubeziehen.

Von den alten Lexika kennt nur Lönnrots Wörterbuch lukka, wofür drei Bedeutungen angegeben werden: 1) 'lingon' 2) 'undergräfven jordkant (vid sandtägt o.s.v.)'; mies oli lukan alla; hauta kaivettiin lukalle 'undergräfdes vid sidorna'; kaivettiin lapsen arkulle sija lukan alle; 3) 'kupa öfver en gaslampa'. Die an zweiter Stelle angeführte Bedeutung stimmt deutlich mit der im SKES angegebenen überein, sogar die Beispielsätze sind dieselben. Lukko begegnet in den alten Wörterbüchern zum ersten Mal eigentlich in den Ergänzungen, die Henrik Gabriel Porthan gegen Ende des 18. Jahrhunderts in seinem Exemplar von Juslenius' Wörterbuch eingetragen hat. Juslenius führt lediglich lukku 'sera; lås' auf, Porthan aber hat an dieser Stelle "Lukko djup grop i en sjö" und "Luco en djup och liten däld. Tav.". Das letztgenannte luko 'Schlucht', das Porthan also mit kurzem $k$ schreibt, kommt s.E. in den Dialekten von Häme (Tawastland) vor. Ganander wiederholt in seinem Wörterbuch nur luko: "Luko en djup och liten däld". In den lediglich handschriftlich überlieferten Ergänzungen zu Juslenius' Wörterbuch (gegen Ende des 18. Jahrhunderts) führt Juhana Canstrén "lucko fovea profundior; antrum; göl; pöl; djup dal" auf. Canstrén, ein Geistlicher aus Pälkäne (Gemeinde in Häme) (s. 
Juslenius lis. III), kannte lukko 'Schlucht' sicher aus seiner Heimatmundart, wo es immer noch sowohl als Appellativ wie auch in Ortsnamen vorkommt. Aber nach Canstrén bedeutet lukko nicht nur 'fovea profundior' oder 'djup dal', sondern auch 'antrum' und 'göl, pöl'. Die letztgenannte Bedeutung erscheint auch in Renvalls Wörterbuch, wo luko (hier einfache Tenuis) 'vallis minor profunda, stagnum; kleines Thal, Teich' ist. Auch Renvall fügt Tav. hinzu, d.h. das Wort kommt in den Mundarten von Häme vor. Bei Eurén bedeutet luko 'liten djup däld; damm', bei Lönnrot 'liten djup däld; fördämning'. Auch z.B. Ahlman führt luko auf, als Bedeutung gibt er 'djup, sank däld; dam' an. Nach Porthan und Canstrén erscheint die heutige Form lukko erst wieder in Lönnrots Supplement: 'fåra, skåra, inskärning, ingröpning, djup dal'. Diese Bedeutungsangaben stimmen fast mit den im SKES für lukko angegebenen Bedeutungen überein.

Von den fi. Wörtern lukka / lukko / luko hebt sich das auf - a auslautende Wort lukka'überstehender Rand einer Sandgrube od. eines Grabes' hervor. Um ähnliches geht es bei der dritten Bedeutung von Lönnrots lukka 'kupa öfver en gaslampa'. Lukko und luko scheinen Varianten eines Wortes zu sein; aufgrund der obigen Ausführungen sind 'Schlucht in Landrücken, Tal, Niederung, Grube, Aushöhlung, Furche, Einkerbung, Rinne, Pfütze, Teich, Damm' Bedeutungen dieses Wortes. Nach den Belegsammlungen des Wörterbucharchivs der fi. Mundarten (SMS) ist die Hauptbedeutung 'tiefe Schlucht, Vertiefung od. Tal in Landrücken', andere Bedeutungen sind: 'grosse grasbewachsene Grube vor dem Haus, Überreste einer Wohnung' (in der Gemeinde Hyvinkää) und 'grosser Graben mit Wasser auf dem Boden' (in der Gemeinde Tyrväntö). Lukko 'Schlucht in Landrücken', das in der Volkssprache sowohl als Appellativ wie auch in Ortsnamen (NT) immer nur mit langem $k$ vorkommt, begegnet in den Mundarten von Zentral- und Süd-Häme sowie von West-Uusimaa. In den Gegenden südöstlich von Tampere, wo Landrücken vorkommen, bezeichnet lukko grosse Vertiefungen in den Landrücken, die nach der Eiszeit entstanden sind. In der Arbeit "Tampereen seudun geologia (Geologie der Gegend von Tampere)" werden sie wie folgt beschrieben: "Stellenweise weist der Landrücken 20-40 m tiefe, steil abfallende Vertiefungen auf, die lukko heissen. Die bekanntesten befinden sich in den westlichen Teilen von Kangasala. 
Ähnliche Vertiefungen mit steil abfallenden Hängen kommen auch in Ylöjärvi vor; in einer dieser Vertiefungen liegt u.a. der Julkujärvi-See. Der Gletscher führte während der Eiszeit Kies und Sand mit sich, die mit dem Stillstand des Eises zu Landrücken angehäuft wurden. Unter diesem Kies wurden vom Gletscher stammende Eisbrocken begraben. Als diese Eisstücke später schmolzen, stürzte der Kies in die Hohlräume hinein, so dass Vertiefungen mit schroff abfallenden Wänden entstanden." (Übersetzung frei nach Matisto - Virkkala 1964 95.)

An. lok, das Koivulehto mit fi. lukko 'Schlucht' vergleicht, bedeutet nach Jan de Vries (1962 364) ausser 'Schluss, Ende' auch 'erhöhtes Halbdeck auf dem Vorderteil eines Schiffes'; jüngere Formen sind isl., fär. und norw. lok, schwed. lock und dän. laag 'Deckel'. Vries weist in diesem Zusammenhang auf an. lúka 'schliessen, öffnen; einhegen; abmachen, bezahlen', lokka 'abziehen, lösen' und lokr 'Kleidungsstück, Rock', eig. 'das Abschliessende, Bedeckende' hin. Nhd. und mhd. Loch, ahd. loh 'Verschluss, Gefängnis, verborgener Aufenthaltsort, Höhle, Öffnung' sind etymologische Entsprechungen von an. lok (Kluge - Mitzka). Etymologisch handelt es sich auf der germ. Seite um dieselbe Wurzel wie bei dem Wort, das als Original für lukko 'Schloss' vorgeschlagen worden ist. Diese germ. Wortgruppe umfasst semantisch Begriffe des 'Schliessens' und ausserdem besonders in den nordgerm. Sprachen Begriffe des 'Bedeckens', denen auf der fi. Seite am ehesten das von Lönnrot genannte lukka 'undergräfven jordkant' oder 'kupa öfver en gaslampa', weniger gut dagegen 'Schlucht, Vertiefung in Landrücken' entsprechen könnte.

Das fi. Wort lukko 'Schlucht in Landrücken, Tal, Vertiefung, Grube, Höhle, Furche, Nut, Rinne' würde ich eher mit an. slok vergleichen; nach Vries 1962 517: an. slok 'Wasserrinne', isl. slokk 'tiefe Stelle im Boden', norw. slok 'Mühlenrinne', schwed. dial. slåk' 'grosser Trog'. Unter diesem Lemma werden ausserdem z.B. folgende Wörter genannt: norw. slük'Abgrund', norw. dial. sløykja 'rinnenartige Vertiefung', vgl. schwed., norw. slūka und dän. sluge, die etymologisch und semantisch dem dt. Verb schlucken entsprechen (Vries a.a.O., s. auch Vries 1971651 s.v. slokken, Kluge - Mitzka). Wörter, die Schlund, Rachen, Kehle usw. bezeichnen, kommen bekanntlich oft als geographische Namen, insbesondere als Namen von Vertiefungen und Schluchten vor (s. 
z.B. Jonsson 1966 145, mehrere Beispiele nennt auch Olsson 1959 85 - 87); vgl. auch fi. juopa 'in der Wassermühle: aus Planken gemachter Trog, der das Wasser in das Wasserrad führt; Mündungsarm des Flusses; Kluft, Schlucht' (Vuorela 1979), ursprünglich 'trinkend', Partizipform des Verbs juoda 'trinken' (s. auch SKES I 126, Hakulinen 1979 200). Mit dem norw. slok (germ. *sluka) verbindet Torp auch norw. dial. sloka 'lang fordybning, rendelignende hulning' und dän. jyll. sloke 'liten dalkløft'. Die germ. Wortgruppe hat also deutlich die für lukko typischen Bedeutungen 'Vertiefung, Tal, Furche, Rinne'. Die Bedeutungen 'Pfütze, Teich, Damm', die in alten fi. Wörterbüchern genannt werden, kommen in der germ. Wortgruppe jedoch nicht vor. Diese Bedeutung kann sich im Finnischen sekundär aus 'Vertiefung, tiefe Stelle' entwickelt haben, auf ähnliche Weise wie Namen vieler Wasserstellen in den nordischen Sprachen (s. näher Jonsson 1966 $300-329,361)^{1}$

Lautlich wäre urgerm. und urnord. *sluka durchaus denkbar als Original von lukko: fi. $l$ - $=$ germ. $s l$-, vgl. z.B. fi. linko 'Schleuder' $\sim$ germ. ${ }^{*}$ slingwōn (SKES, Koivulehto 1973 11), fi. $-k k-=$ germ. -k-, vgl. z.B. fi. sakko 'Busse' $\sim$ germ. * sakō (SKES), zum auslautenden Vokal s. z.B. Hakulinen 1979 175. Die Tatsache, dass lukko nur in fi. Dialekten von Häme vorkommt, sollte kein Grund zum Zweifel an der Entlehnung sein. Ein solches altes germ. LW ist auch z.B. fi. verkilö 'Knopfloch, Öse, Aufhänger' ( urgerm. *wergila, vgl. an. virgill 'Strick, Schnur'), das ebenso in Häme begegnet (SKES VI 1706 - 1707; die s.v. verkilö genannten estn. Wörter gehören vielleicht nicht zu dieser Wortgruppe). Das Verbreitungsgebiet von verkilö deckt sich eigentlich mit dem von lukko.

\section{2. kulppa, kulppi}

Ähnliche semantische Komponenten wie bei der Wortgruppe lukko liegen zum Teil auch bei den in SKES (II 235) unter dem Stichwort kulppa angeführten Wörtern vor. Fi. kulppa ist 'eine Grube, die sich mit Wasser gefüllt hat, Pfütze, Moortümpel, kleine

${ }^{1}$ Vgl. jedoch auch schwed. dial. fluk 'mindre vattensamling, pöl (på väg); utvidgning av vattendrag', flucka 'liten vattensamling, pöl, vattenhål, regnpuss' (Jonsson 1966346 - 347) und flok, floke, floka (345-346), lok, lök 'Pfütze' (269 - 270). 
grubenartige Vertiefung z.B. im Fluss od. See' (diese dem SMS entnommenen Bedeutungen begegnen in erster Linie in den südöstlichen Mundarten; vgl. auch Nissilä 1962 59: 'Bucht, bei Ebbe ein Teich' und Nissilä 1975 32: 'kleiner Teich, Pfütze') und 'Vertiefung im Schnee, die dadurch entstanden ist, dass Wasser von der Traufe tropft' (diese Bedeutung in den Dialekten von Häme und Satakunta); zu der letztgenannten Bedeutung gehört auch das kulppa des westfinnischen Kalendersprichwortes "Kun kukko sipinpäivänä kulpasta juo, niin maarianpäivänä isosta allikosta" ("Wenn der Hahn am Tag des Siegfried aus dem kulppa trinkt, so zu Mariä Verkündügung aus der grossen Lache"; Tag des Siegfried 15.2., Mariä Verkündigung 25.3., s. Kuusi 1953205 und K. Vilkuna 196842 - 43, 65 - 70).

Lönnrot übersetzt kulppa mit 'vattupöl' und kulpanne mit 'grop, djupt ställe (i åar)' (kulpanne lt. Dialektbelegen (SMS) nur in der Gemeinde Hausjärvi, wo es 'die Stelle im Wasser, wo untief plötzlich tief wird' bezeichnet). Estn. kulbas (Gen. Sg. kulpa) bedeutet 'ausgehöhlter Uferwall, Loch im Uferwall, Druckstelle, Delle' (SKES, über die estn. Bedeutungen von kulbas s. noch Saareste I 214 - 215, 778 - 779, II 658 und Pall 1969 201). Gruben und Unebenheiten, oft in Verbindung mit Wasser, sind typische Bedeutungsinhalte dieser fi.-estn. Wörter.

Zweitens gibt das SKES für fi. kulppi die Bedeutung 'Beule (im Schuh)' ('Delle, Beule, Knick od. Falte im Stoff od. am Schuh'; SMS) an; dem entspricht, was die Bedeutung betrifft, karel. kul'p$p u$ 'Blase' und kul'ppa 'beulig, ausgebeult, von unordentlichen Stellen in Kleidungsstücken, von ungleichmässigen Falten' sowie kul'ppusilmä 'einer mit hervorstehenden Augen, Glotzauge' (KKS).

Drittens bedeutet fi. kulppi, kulppa, kulppo 1t. SKES 'Schüssel, Becher, Kelle' (in den Mundarten nur kulppi; SMS); wot. kulppi bedeutet 'Kelle' (SKES), estn. kulp (Gen. Sg. kulbi) 'Schöpflöffel, Schöpfkelle' (EDWb). Diese Bedeutung, die in drei osfi. Sprachen begegnet, nennt das SKES an erster Stelle. Es wäre in der Tat möglich, die Bedeutung 'Kelle' als primär anzusetzen und die weiteren als sekundäre Entwicklungen zu erklären. In den skand. Sprachen gibt es jedoch mehrere Wörter, die eigentlich nicht ausser acht gelassen werden dürfen, wenn man die osfi. Wortgruppe kulppa, kulppi untersuchen will. 
Im SKES wird bei kulppa gefragt, ob norw. kulp 'Wassergrube, Pfütze' möglicherweise aus dem Finnischen stamme. Dieses norw. Wort wurde später von Hans Jonsson in seiner Abhandlung Nordiska ord för vattensamling (1966 318 - 319) behandelt, ohne die Überlegung im SKES zu berücksichtigen; es sei bemerkt, dass Jonsson das SKES sonst (z.B. was die fi. Wörter kuru, kulju und pade betrifft) wohl zu Rate gezogen hat. Das Wort begegnet nach Jonsson in mehreren norw. Mundarten: kulp 'fördjupning i botten av vattendrag (eller vattensa mling)' (Østlandet, Hordaland, Nordland), kulp (auch vasskulp) 'liten tjärn eller vattenhåla o. dyl.' (Vestfold, Buskerud, Telemark, Oppland, Østfold, Hedmark); in Buskerud enden Namen mehrerer kleiner Teiche auf -kulp(en). Aber das Wort begegnet auch in den schwed. Mundarten: kulpa '(mindre) fördjupning i marken, fördjupning i väg' (Småland, Närke, Västmanland), 'bråddjupt ställe i en mosse, en sjö eller ett vattendrag' (Värmland), 'fördjupning i sjö' (Småland); Kulpan begegnet vielerorts in Nordmark (Värmland) als Name kleiner Seen. Dieser Name beruht lt. Jonsson auf der appellativen Bedeutung 'vattenfylld fördjupning, (liten) tjärn el.dyl.'. Nach dem Zeugnis solcher in Skåne vorkommenden Ortsnamen wie Kulpen, -kulpen, Kulparna, -kulparna scheint kulp 'fördjupning i terrängen, grop el.dyl.' früher auch in Südschweden produktiv gewesen zu sein.

Schwed. und norw. $k u l p(a)$ wurde etymologisch in erster Linie mit den schwed. Wörtern köl und kälke (norw. kjøl, dt. Kehle) verbunden; die Grundbedeutung wäre in diesem Falle also ebenfalls 'Schlund, Rachen' (Jonsson 1966 319). Sowohl lautlich als auch semantisch könnte schwed.-norw. $k u l p(a)$ mit fi.-estn. kulppa, kulbas '(Wasser-)Grube' verglichen werden. Die weite Verbreitung des skand. Wortes und sein häufiges Vorkommen in Ortsnamen spricht für die Annahme, dass das Wort aus dem Skandinavischen ins Ostseefinnische, und nicht umgekehrt, entlehnt wurde.

Bei den Wörtern, die im SKES unter kulppa behandelt werden, scheinen die Bedeutungen 'konkav' und 'konvex' zusammengefallen zu sein, da fi.-karel. kulppi, kulppa auch 'Beule, Blase' sein kann. Dies ist an sich nichts Aussergewöhnliches, z.B. fi. nikama kennt sowohl die Bedeutung 'Furche, Nut' als auch 'Erhebung' (SKES II 379). Ebenfalls im SKES (IV 872 - 873) wird für fi. ruopas 'Stein- od. Eishaufen' die weps. Entsprechung roppaz 'Loch, Vertiefung (z.B. im Weg)', rophik 'Graben, Loch (in der 
Erde, im Weg)' angegeben. Interessant ist die Tatsache, dass auch auf skand. Seite Wörter zu finden sind, die mit kulp-verwandt sind und denen die Bedeutung 'gerundet' oder 'konvex, gebeugt, gewölbt' eigen ist: isl. kúlpeyg $ð$ 'det at have udstaaende Øjne' (Blöndal) (vgl. karel. kul'ppusilmä 'einer mit hervorstehenden Augen'), norw. dial. kulp 'kort, næsten valseformig Tingest', kolp 'fremtand paa ku' (Torp), schwed. dial. $k u l p(a)$ 'ovig, lunsig person', kulpig 'välvd; knölig, bukig' (Jonsson 320).

Schwed. kulpig bedeutet andererseits auch 'inböjd; skålformigt urtagen; urholkad' (Jonsson 320), was an die osfi. Bedeutungen 'Kelle, Schale' erinnert. Kulppi in dieser Bedeutung möchte ich jedoch mit einer anderen germ. Wortsippe vergleichen. Als Original käme die Wortsippe in Frage, die durch norw. dial. skolp 'liten uthulet blok el. træflaske til brynet' (Torp), schwed. skölp 'urgrävning' (Hellquist), dän. skulpe 'torummet kapsel', skylp, skølp 'hulning' (Nielsen) und weiter z.B. in mhd. scholpe, schulpe und nl. schulp 'Muschelschale' (Vries 1962 s.v. skolpr und Vries 1971 s.v. schelp) vertreten ist. Die urnord. Form wäre *skulp-; lautlich vgl. z.B. fi. kuuro 'Regenschauer' germ. *skürō (SKES II 252) und fi. helppo 'leicht' germ. *helpō (SKES I 67 - 68, Koivulehto 1974 122). Das osfi. $-i$ kann als Ableitungssuffix gedeutet werden (so Hakulinen 1979 121). Die Annahme, dass ein Wort wie kulppi aus dem Urnordischen entlehnt worden sei, könnte auch dadurch unterstützt werden, dass (os)fi. malja 'Schale, Schüssel, Becher' (SKES II 330, Koivulehto 1978310 - 311) und (os)fi. kippa 'Trinkgefäss, Schale' (SKES I 197, Raun 1971 75) ebenfalls germ. LW sind.

\section{3. hule}

Das SKES behandelt hulas (u.a. 'Ecke, Winkel'), hule (in hulejy$v \ddot{a}$ 'leeres, leichtes Korn') und hulhava (z.B. mennä hulhavalle 'verlorengehen') getrennt nacheinander. Für hulas und hulhava wird eine (mit einem Fragezeichen versehene) germ. Etymologie angegeben. Als Original wird in beiden Fällen germ. *hul- vermutet, das für hulas in an. holr 'hohl, von innen leer' und hol 'Loch, Bauchhöhle' und für hulhava in ae. holh 'Höhle, Aushöhlung, Kluft' und ahd. hulwa 'Teich' (SKES I 85) vertreten ist. (Zu hulas und hulhava s. auch Toivonen 1944348 - 352 und SKES V 1526 - 1527 s.v. ulappa. Für hulhava hat Osmo Mäkeläinen, 197566 - 
74 , ein anderes germ. Original vorgeschlagen, und zwar *uulh(a)$w a-$, vgl. schwed. ulv, dt. Wolf; diese Gleichung ist jedoch mit lautlichen und semantischen Schwierigkeiten verbunden.) Eher noch als hulas und hulhava scheint mir das unberücksichtigt gebliebene hule auf die oben genannte germ. Ausgangsform zurückzuführen zu sein.

Hulejyvä ist nach dem SKES also 'leeres, leichtes Korn' (-jyvä 'Korn'), vgl. Lönnrot: 'ett kärnlöst, tomt korn'. Toivo Vuorela (1979 82) definiert huleet (Nom. Pl. von hule) wie folgt: 'die minderwertigen, leichten Körner, die beim Worfeln näher beim Worfler herunterfallen als die besseren und schweren, esilliset, hulmut, hulut, humeet, humelot, humut, husut, kahat, rajaiset'. Unter diesen von Vuorela aufgeführten Synonymen gibt es mehrere Wörter, die lautlich eine Ähnlichkeit mit hule haben, so dass auch hule leicht mit zu den deskriptiven Wörtern "geworfelt" werden könnte. Es ist jedoch offensichtlich, dass die genannten Nebenformen grösstenteils unter dem Einfluss der semantisch ähnlichen Wörter kul$m u$ und kumu entstanden sind: fi. kulmut (Nom. Pl.) 'Heu- od. Futterkrümel, Ausputz', karel. kulmu, l'iinankulmut, kulmus 'Deckblättchen vom (Flachs-)Samen' (SKES, vgl. KKS: karel. kulmu 'leere Samenkapsel (meist vom Flachs)' in der Gemeinde Uhtua); fi. kumu ist z.B. nach Nirvis Kiihtelysvaaran murteen sanakirja ("Wörterbuch des Dialekts der Gemeinde Kiihtelysvaara") 'Schale und Ausputz beim Trennen des Flachssamens vom Kerngehäuse; oberste Blätter des Flachses, Gehäuse und Samen, die beim Aushülsen abfallen'.

In den fi. Dialekten wird das Wort hule meist von Haferkörnern gebraucht. Am weitesten ist hule in den südöstlichen Mundarten und in den östlichen und teils auch in den südlichen Savo-Dialekten verbreitet, aber es wurde auch auf dem Gebiet der westfinnischen Mundarten in Satakunta (in Kokemäki, Punkalaidun, Karvia und Kihniö) aufgezeichnet (SMS). Bei hule weist das SKES auf nordöstl. estn. Dial. (möglicherweise dem Finnischen entlehnt) huled, hulemed (Nom. Pl.) 'leichte Körner, die beim Worfeln neben dem Haufen bleiben'. In diese Wortgruppe gehört m.E. auch karel. (in den Gemeinden Korpiselkä und Suojärvi) hulakka (Adj.) 'schlechte Ähren tragend, taubährig', wofür das KKS den Beispielsatz: "aiga on Jumalan vil'l'ad hulakat tänä vuoden, yhed on aganazed vai" ("taub sind die Ähren in diesem Jahre, lauter 
Kornschalen") anführt.

Hule ist bisher wahrscheinlich deshalb etymologisch nicht weiter untersucht worden, weil das Wort irgendwie mit fi. hulahtaa 'plumpsen, platschen' (FDGWb) verbunden worden ist. Dieses Verb kann man ja z.B. nach NS auch "von plötzlichem Fallen, Rutschen oder einer ähnlichen Bewegung" benutzen. Somit wäre die Bezeichnung huleet (Nom. Pl.) gerade auf die Bewegung beim Worfeln zurückzuführen, ähnlich wie etwa fi. vareet (Nom. Pl.; Nom. Sg.: vare $^{2}$ ) 'abgefallene Krümel, Abfälle, Ähren, Körner, Heu' o.dgl. offenbar mit fi. varista 'abfallen' zusammenhängt (SKES V 1655). Das NS nennt für huleet und hulejyvät die Synonyme kahut resp. kahujyvät, die wiederum lautmalerisch auf dem Rascheln (kahista) dieser Körner beruhen dürften; auch kahu ist nach dem NS 'Rascheln' (s. auch Hakulinen 1979 222).

Aber wenn man im Auge behält, dass es sich bei hule um ein 'leeres, hohles Korn' handelt, könnte man es mit dem oben genannten an. holr 'hohl' vergleichen, dessen urgerm. Form *hula- ist und das gleichbedeutende Entsprechungen in den anderen nordischen Sprachen hat (s. Vries 1962 248, Nielsen s.v. hul). Plausibler wäre es jedoch, von einem germ. Substantiv auszugehen, was auch

${ }^{2}$ Das zweite fi. vare 'Grabruine, Hünengrab' (NS), 'Steinhaufen' (SKES V 1652), dem im Estnischen vare (Gen. Sg. vare) 'Steinhaufen', vare (Gen. Sg. vareme) 'Ruine, Trümmer' (EDWb) entspricht, könnte m.E. wiederum ein germ. LW sein. Man vergleiche es mit an. vor 'Landungsstelle', eigtl. 'am Landungsplatz aufgeschichtete Steine' (Vries 1962 674) und mit norw. vor 'høi banke av sten el. grus; rad av stener paa hver side av en stød' (Torp). Urnord. *waru- ist in einer in Norwegen gefundenen Runeninschrift (von ca. 500) belegt: waruR 'Steinhege, Steinhegung (um das Grab)' (Krause 1971 50, 168). Diese Bedeutung führt das urnord. Wort in die Nähe der von Menschenhand errichteten Steinschichtungen entlang der Küste, die mit dem fi.-estn. vare bezeichnet werden (zu vare s. genauer z.B. K. Vilkuna $196595-99$, Nissilä 1967298 - 299, T. Itkonen 1972105 und Vahtola 1980294 - 296). Das Verhältnis fi. vare $\sim$ urgerm. ${ }^{*}$ waruz ist vergleichbar z.B. mit fi. luode 'Nordwest; Ebbe' urgerm. flōđuz. Erst in späteren Entlehnungen kommt im Finnischen auch -us vor (vantus, palkus); dieser Typ (-us: -uhu- $>-\bar{u}-)$ ist im Finnischen sehr selten (vgl. T. Itkonen 1973a 54; auch urfi. *-tt- und *-kk-in *vanťtus und *paľkkus beweisen, dass diese Wörter nicht zu den ältesten germ. LW im Finnischen gehören). Auf der semantischen Seite kann vare mit fi. raunio 'Steinhaufen, Ruine' (estn. raun, Gen. Sg. rauna, 'Steinhaufen, steinige Stelle') verglichen werden. Raunio ist für ein germ. LW gehalten worden, vgl. an. hraun 'steiniger Boden, Lava', dän. røn 'Steinbank auf Meeresboden' (Vries 1962 252, s. auch SKES III 749, Hakulinen 1979 362, auf Seite 363 gibt Hakulinen für raunio das Synonym vare an). 
aus semantischen Gründen näherliegt. Für den Vergleich eignet sich dabei am besten ae. hulu 'Hülse, Schale' (Holthausen), engl. hull< urgerm. *hulo (Seebold 1970 252). Zu derselben Wurzel, die nach Seebold (a.a.O.) in urgerm. *hela- 'verbergen' vertreten ist, wird auch dt. Hülse gestellt. Germ. LW im Finnischen mit $-e$ für germ. ${ }^{*}-\bar{o}$ ist z.B. das semantisch hule nahestehende helve 'Spelze, Granne' ( urgerm. *helwō(n)).

Die germ. Herkunft von hule scheint auch aus dem Grunde möglich, weil semantisch sehr nahestehende Wörter aus dem Germanischen entlehnt worden sind. Ausser dem oben genannten helve (s. z.B. SKES I 68) gehören folgende Wörter zu dieser Gruppe: akana 'Granne, Spreu' (SKES I 13), kale 'Hüllblatt der Ähre, taubes Korn, Granne' (SKES I 146-147; kann auch auf urgerm. *-ō: *skalō zurückgeführt werden, vgl. ae. scealu 'Schale, Schote' (Holthausen) und schwed. skal), kauna 'Spelze, Granne' (SKES I 173) und pahna 'Stroh, Streu' (Hofstra 1980).

\section{4. keikka und keno}

Nach dem NS begegnet keikka in der heutigen fi. Standardsprache einerseits in Lokalkasus mit adverbieller Funktion: keikassa 'schief, rückwärts geneigt, gebeugt', keikallaan, keikalleen 'schief, schräg, geneigt'; andererseits begegnet dieses Wort in $\mathrm{Zu}$ sammensetzungen: keikkaniska 'den Hals nach hinten gebeugt', keikkanokka 'Stupsnase', keikkaselkä 'Hohlrücken'. Ausserdem kommt keikka in der Zusammensetzung kuperkeikka 'Purzelbaum' vor. Keikka 'nach hinten gebeugt' begegnet in alten Wörterbüchern und in Dialekten; lt. SKES (I 177) verfügt es über die gleichbedeutenden Nebenformen keikko, keikku und keikas; ferner z.B. in Wörterbüchern von Lönnrot und Eurén keikki 'upprätt 1. utsträckt ställning' und in den Wörterbüchern von Lönnrot und Ahlman keikkeä 'som bär hufvudet upp, uppsträckt, sprättaktig' (Lönnrot), 'bakåtböjd, sprättaktig' (Ahlman). Nach Juslenius bedeutet keikka 'recurvus, sursum curvatus; krökt uppåth'; Ganander nennt in seinem Lexikon ausser diesen Bedeutungen auch die Bedeutung 'slädmede', die in der Tat in den fi. Dialekten noch heute als Bedeutung von keikka vorkommt. Keikka kann nämlich '(gebogene) Kufe eines Schlittens, einer Wiege od. eines Schaukelstuhls' bedeuten (Vuorela 1979). Fi. keikka hat eine Entsprechung im Karelischen: keikkapeä 'jemand, der den Kopf stolz 
hoch trägt' und keikkaselkä 'jemand mit einem Hohlrücken' (KKS). Die Bedeutung 'Kufe' scheint sich im Finnischen entwikkelt zu haben und beruht auf der für eine Kufe typischen gebogenen Form, vgl. Lönnrots Beispielsatz: reen nokka on ylöspäin keikassa'der Vorderteil des Schlittens ist hochgebogen' (s.v. keikka).

Das SKES hält keikka für eine mögliche Entlehnung und vergleicht es mit an. keikr 'nach hinten gebeugt (vom Oberkörper)' und norw. keik 'Falte, Kurve, Schiefheit; Verstauchung, Verrenkung'. Die Bedeutung 'schwingend, schaukelnd' für fi. keikka, die namentlich bei zahlreichen Verben vorliegt, sollte nach dem SKES möglicherweise hiervon getrennt gehalten werden: "vielleicht sind im Finnischen zwei verschiedene Wörter zusammengefallen". Solche fi. Verben sind z.B. keikata 'schwingen, schaukeln', keikahtaa 'sich schnell umdrehen', keikota 'id.', keikuttaa 'schwingen, schwanken (machen), schwankend gehen', keikkua 'sich von einer Seite zur anderen bewegen, schwingen' (NS). Zu der letztgenannten Wortgruppe keikka 'schwingend, schaukelnd' gehören auch estn. kõigutada 'schaukeln, schwingen, hin- und herbewegen; wankend machen, schwankend machen, zum Wanken bringen; schütteln, rütteln, erschüttern' und kõikuda 'schwanken, wanken, wackeln; unschlüssig (unentschlossen) sein' (EDWb); bei Wiedemann auch kõik (Gen. Sg. kõigu) 'Schwanken, Schwankung, Erschütterung'. Im Karelischen begegnet keikkuo 'schwanken' und keikottaa, was sowohl 'schief, geneigt sein' als auch 'schwanken machen' (KKS) bedeutet. Im Ingrischen kommt keikkua 'schwanken' (IMS) vor. Zu dem Verb *keikkā : keikkab in E. N. Setäläs Aufzeichnungen des Wotischen s. MSFOu 135 64 . Es sieht tatsächlich so aus, als sei diese Wortgruppe semantisch nicht mit an. $k e i k r$ oder mit dem entsprechenden an. Verb keikja 'rückwärts beugen' (Vries 1962 304) zu verbinden; mit dem Altnordischen könnten nur das obengenannte karel. keikottaa 'schief, geneigt sein' und fi. keikata, das lt. Lönnrot auch 'böja sig bakåt, högfärdas, sprättas; böja utåt, krumma' bedeutet, zusammenhängen.

Dagegen lässt sich die Wortgruppe keikka 'schwingend, schaukelnd', die weiter als die erstgenannte Wortgruppe keikka 'nach hinten gebeugt' verbreitet ist und offensichtlich auf einem Verb basiert, m.E. gut mit dem an. Verb skeika 'schwanken, schlingern' (Vries 1962 488), 'unsicher gehen, schwanken, von der Richtung abweichen' (Baetke) vergleichen. An. skeika ist verwandt mit isl. 
skeika 'slingre til Siden, forlade den rigtige Retning' (Blöndal), norw. skeika 'skeie, vige ud fra den rette Linie, gaae en skjæv eller kroget Gang' (Aasen), schwed. dial. skeka 'skreva (med benen)' (Hellquist) und dän. skeje 'som udtryk for (skæv) bevævelse ell. retning til siden (bort fra en lige linie olgn.)' (ODS). Semantisch wie auch lautlich könnte das osfi. Verb keikata, keikkua usw. mit der an. Form verbunden werden, lautlich vgl. z.B. fi. keidas 'Landenge' $\sim$ an. skeið (T. Itkonen $196255-56$ ). Wegen der weiten Verbreitung des Wortes im Ostseefinnischen muss jedoch auch die Möglichkeit einer früheren Entlehnung in Erwägung gezogen werden. In dem Falle wäre urgerm. und urnord. *ai- (*skaikōn-) im Ostseefinnischen durch - ei- wiedergegeben worden, ähnlich wie z.B. bei fi. keihäs 'Speer' urgerm. *gaizaz (SKES I 177). Dieses Verb, das eine Bewegung zum Ausdruck bringt, hat sich im Ostseefinnischen sicherlich schon früh den einheimischen $u$-Ableitungen angeschlossen (der Stamm keikku- begegnet im Finnischen, Karelischen, Ingrischen und Estnischen; zu den $u$-Ableitungen s. Hakulinen 1979292 - 293 und Laanest 1975 188). ${ }^{3}$

Als ein Bedeutungsäquivalent für die Form keikassa nennt das NS ein anderes, im Lokalkasus verwendetes Wort, und zwar kenossa, kenolla(an) 'rückwärts geneigt oder gebeugt', z.B. pää / niska / kaula / selkä kenossa'den Kopf / den Nacken / den Hals / den Rücken nach hinten gebeugt', auch: etukenossa 'vorwärts gebeugt' und takakenossa 'rückwärts gebeugt'. Nach dem NS sind keikkaniska und kenokaula gleichbedeutend. Von den anderen osfi. Sprachen kennt das Estnische das Wort keno in kenus (Inessiv Sg.) 'stolz nach hinten gebeugt'. Dieses estn. Wort, das verbreitet in den westlichen estn. Dialekten vorkommt (s. Saareste 1953 15), könnte nach dem SKES (I 181) vielleicht aus dem Finnischen entlehnt sein. Das SKES gibt für keno Entsprechungen aus den

${ }^{3}$ Mit germ. *skaik- (> an. skeika) ist auch norw. skeina 'flyve skjævt el. med avvekslende helding til siderne, som en plate i luften; glide av til siden' (Torp, s. auch Hellquist s.v. skena ${ }^{1}$, Nielsen s.v. skeje) verwandt. Es handelt sich um unterschiedliche Ableitungen von der idg. Wurzel *skĕ̈i- (s. Nielsen a.a.O.). Das norw. Verb könnte mit dem fi. Verb keinua 'schaukeln, schwingen, schwanken, schlingern' (FDGWb), 'gunga, svänga sig' (Lönnrot), 'oscillo jactor; gungar' (Juslenius) verglichen werden. Hakulinen (1979 293) stellt fest, dass die Herkunft von keinua nicht geklärt ist. Das Wort keinu 'Schaukel' wird von Hakulinen (1979 223) bei den deverbalen Nominalsuffixen behandelt. 
fiu. Sprachen ausserhalb des Ostseefinnischen an, hält diese jedoch alle für unsicher. Die aufgeführten Wörter bedeuten meist 'auf dem Rücken', lediglich eine der ostj. Entsprechungen hat die Bedeutung 'nach hinten gebeugt'. Keno kann also als ein osfi. Wort behandelt werden.

Fi. keno hat auch eine andere, allerdings weniger bekannte Bedeutung. In seiner Untersuchung des von Anders Wikman (einem Geistlichen aus dem Kirchspiel Längelmäki in Mittelhäme) verfassten, handschriftlich überlieferten Wörterbuchs aus der ersten Hälfte des 19. Jahrhunderts behandelt Martti Rapola $(1956$ 94, 95) zwei in dieser Handschrift vorkommende Bootstermini, von denen nur das letztere in den Wörterbüchern genannt wird. Es handelt sich um die Wörter keno und kinu. Nach Wikmans Erläuterung bedeutet keno 'stam, kinu, perä (venheisä)' (perä venheisä 'Heckteil des Bootes'), kinu wiederum 'stäf, stam, keula, keno, perä (venheisä)' (keula 'Vorderteil des Bootes'). In den Mundarten sind keno und kinu in erster Linie in Häme bekannt, wo sie 'Bug, Vorderteil des Bootes', auch 'hochgebogener Vorderteil des Schlittens' bedeuten (Rapola a.a.O.). ${ }^{4}$

Kinu ist nach Euréns Wörterbuch dasselbe wie fi. kokka, das er mit 'något uppstående, spetsen af fram- och bakstammen på en båt' ins Schwedische übersetzt. Nach Lönnrot ist kinu 'båtens krokiga fram- och bakstams trä'. Das NS, das kinu als dialektal bezeichnet, gibt dafür - wie auch das SKES s.v. kinunki - die Bedeutung 'Steven' an. Toivo Vuorela (1979) erläutert kinu wie folgt: 'Verlängerung des Kiels im Bug oder Heck, Steven'. Hierzu dürfte auch das in Südostbottnien vorkommende kino 'Bandeisen eines Wagenrandes' gehören, vgl. auch kokkakino 'Bug des Bootes' in Mittelfinnland (Vuorela 1979).

Die ursprüngliche Bedeutung beider oben erläuterten kenoWörter könnte 'hochgebogener Vorderteil (od. Hinterteil) des

\footnotetext{
${ }^{4}$ Eigentlich ist Wikman nicht der erste, der keno mit einem Boot in Verbindung bringt, denn auch H. G. Porthan nennt keno in den Ergänzungen, die er in seinem Exemplar von Juslenius' Wörterbuch eingetragen hat. Nach Juslenius bedeutet keno 'erectus; rak', kenokaula 'extento collo incedens; rakhals' und kenokas 'homo erecto capite; som sätter näsan i wädret'. Porthan fügt den Satz Venhe on kenollansa hinzu und übersetzt kenollansa ins Schwedische mit 'framlastad' (dt.: 'Das Boot ist vorderlastig'). Ein Fragezeichen vor diesem Satz besagt jedoch, dass er, was die Bedeutung angeht, wohl nicht ganz sicher war. Der Beleg kommt seiner Angabe nach jedenfalls aus Häme.
} 
Bootes' sein, wobei keno 'gebeugt' eine übertragene Verwendung von keno 'Bug' wäre. Eine Parallele bildet z.B. fi. koukku 'Haken' in Ausdrücken wie selkä koukussa'einen krummen Rücken haben' und koukkuselkä 'krumm' ('krummer Rücken'). Sowohl für keno als auch für kinu kann eine germ. Etymologie vorgelegt werden. Was kinu angeht, so hat das SKES nebenbei auf diese Möglichkeit unter dem Stichwort kinunki hingewiesen. Fi. kinunki (auch kinnunki) 'Schlagseite des Bootes od. der Schiffsseiten im Bug und Heck' ist für eine Entlehnung aus dem finnlandschwed. dial. kinnungar 'seitlich schief geschnitzte Brettstücke an den Enden der zweituntersten Bandbretter' (auch kinnungs-bord 'zweites Bordbrett über dem Kiel') gehalten worden. "Hierzu vielleicht auch kinu 'Steven' ', stellt das SKES fest. (SKES I 196, s. auch Karsten 1943 - 1944 208.)

An. kinnungr bedeutet 'Schiffsbug; Kerbe am Ende eines Balkens', eigtl. 'Wangengegend', denn der erste Teil des Wortes ist kinn 'Wange', vgl. norw. kinn und schwed., dän. kind (Vries 1962 309). Auch kinn allein begegnet in der Bedeutung 'Bootsteil, Vorderteil des Bootes', vgl. z.B. Fritzner: kinn 'en vis Del af Fartøi (maaske = kinnungr, hlýr ?)' und Heggstad: kinn 'bog (på skip) $=$ hlýr'; beim nhd. Kinn, das etymologisch derselben Herkunft ist, führt Grimms Wörterbuch auf: "im schiffsbau heiszt kinn und kinnback, nl. kin, kinnebak das vordere ende des kiels, woran der vorsteven stöszt, das hintere ende heiszt hiel, hieling, engl. heel, d.i. ferse, wie bei einem schwimmer das kinn und die fersen vorn und hinten vorstehen und im wasser arbeiten". Dass Bezeichnungen der Körperteile auf Teile des Bootes übertragen werden, ist eine bekannte Erscheinung. ${ }^{5}$

${ }^{5}$ Mehrere Beispiele werden u.a. von Falk-Torp s.v. kjelke angeführt. Dieses kjelke gehört zu derselben skand. Wortgruppe, aus der auch fi. kelkka, estn. kelk (Gen. Sg. kelgu) '(Hand-, Rodel-)Schlitten' (s. SKES I 179) entlehnt wurde und ist nach den gebogenen Kufen benannt, vgl. an. kjalki'Kinnbakken; Schlitten' (Vries 1962310 - 311). Gleichen Ursprungs ist an. kjolr 'Kiel, Kielraum; Schiff; Name e. Bergrückens' (Baetke), vgl. auch an. kjalar-hall 'hinterster Teil des Kiels', kjalar-tré 'Baumstamm, der als Schiffskiel dient' (Baetke). An. kjolr wird auf die urnord. und urgerm. Form *kelu-zurückgeführt (Vries a.a.O., Torp s.v. kjøl, Hellquist s.v. köl, Nielsen s.v. køl). Mit urgerm. und urnord. *kelu- wurde fi. kelo verglichen (Setälä 1913383 und die dort genannte Literatur). Die Gleichung hat jedoch als unsicher gegolten; in SKES wird das Wort nicht erwähnt. Kelo begegnet in allen älteren fi. Wörterbüchern (Juslenius, Ganander, Renvall, Helenius), nach denen kelo dasselbe 
An. (und nhd.) kinn wird auf die urgerm. Form *kinnu- zurückgeführt, was wiederum auf die noch frühere urgerm. Form *kenw-/ ${ }^{*} k e n u$ - zurückgeht (Hellquist, Nielsen, Vries, s. auch Vries s.v. kné). Im Zusammenhang mit seiner Behandlung von an. kjanni, kjannr 'Kopf', isl. kjanni 'Kieferbein' und kjannur 'Kinnbacken' äussert de Vries (1962 311) die Vermutung, dass an. kjanni, kjannr auch zu an. kinn gehören könnte; er rekonstruiert die urgerm. Form *kenan, s. auch Jóhannesson 342, vgl. jedoch Jóhannesson 1049. Osfi. keno wäre vor germ. -enC->-inC-, fi. kinu nach diesem Lautwandel entlehnt worden. Auch hinsichtlich des Vokalismus im Finnischen scheint diese Deutung plausibel. Im Ostseefinnischen begegnen sowohl alte germ. LW (z.B. fi. kello, pelto, rehto) als auch alte einheimische Wörter (z.B. fi. hepo, neuvo, vero) mit dem Vokalismus $e-o$ (s. T. Itkonen 1980 113). An diese Wörter hat sich auch das aus dem Germanischen stammende *kenu angeschlossen, da Wörter mit dem Vokalismus $e-u$ unter den ältesten germ. LW nicht vorkommen. Zu den alten germ. LW im Ostseefinnischen gehört bekanntlich eine ganze Menge Wörter aus dem Bereich der Seefahrt (s. z.B. Hertzen 1973 94, Laanest 1975 219, Hakulinen 1979 359); diese Gruppe kann also um keno erweitert werden.

OSMO NIKKILÄ

wie fi. keula 'Vorderteil eines Bootes od. Schiffes' bedeutet. Auch in dem obengenannten Wörterbuch Wikmans wird kelo 'stäf' (Rapola 1956 94) und weiter peräkelo 'bakstam, perä, peräkeula' ('Hinterteil, Heck im Boot') (Rapola 1956 107, dort auch über die dial. Verbreitung des Wortes) erwähnt. Mit dem obengenannten an. kjolr verwandt sind ae. ceole 'Kehle, Schlund, Schlucht; Schiffschnabel' (Holthausen), mnd. und mnl. kele, ahd. kela 'Kehle, Hals' und nhd. Kehle. Diese Wörter sind auf die urgerm. Form *kelōn zurückzuführen (s. z.B. Kluge - Mitzka, Torp); diese Form wiederum könnte "direkt" das Original von fi. kelo sein. - Sollte auch fi. kelo 'ausgedorrte Kiefer', karel. kelo, k'elo, keloi id. (KKS) und lüd. keлo id. (LyMS; SKES I 180) hierzu gehören? Kelo kommt recht oft in Zusammensetzungen vor, z.B. fi. kelopuu (-puu 'Baum'), kelohonka (-honka 'Kiefer') (NS), karel. keloipuu, kelohonga (KKS). Mit dem fi.-karel. kelo(i)puu zu vergleichen wäre an. kjalar-tré. Kelo könnte also aus der Zusammensetzung kelopuu od. kelohonka abstrahiert worden sein; die ursprüngliche Bedeutung wäre etwa 'Baum(stamm), aus dem der Vorderteil des Schiffes gebaut werden kann'. Nach Terho Itkonens Deutung (1973b) sei osfi. mänty 'Kiefer' das Ergebnis einer ähnlichen Ellipse: mäntä 'Quirl' + petäjä 'Kiefer' = *mäntäpetäjä/mäntypetäjä 'Quirlkiefer' $\rightarrow$ mänty 'Kiefer'. 


\section{LITERATUR}

Aasen $=$ Ivar Aasen, Norsk Ordbog med dansk Forklaring. Fjerde uforandrede Udgave. Kristiania 1918.

Ahlman $=$ Ferd. Ahlman, Ruotsalais-suomalainen ja suomalais-ruotsalainen sanakirja. Toinen, lisätty painos. Helsinki 1883.

Baetke $=$ Walter Baetke, Wörterbuch zur altnordischen Prosaliteratur I - II. Berlin 1965, 1968.

Blöndal = Sigfús Blöndal, Islandsk-dansk Ordbog. Reykjavík $1920-1924$.

$\mathrm{EDWb}=\mathrm{K}$. Kann - E. Kibbermann - F. Kibbermann -S. Kirotar, Estnisch-deutsches Wörterbuch. 2. Auflage. Tallin 1970.

Eurén $=$ G. E. Eurén, Finsk-svensk Ordbok. Hämeenlinna 1860 .

Falk - Torp $=$ H. S. Falk und Alf Torp, Norwegisch-dänisches etymologisches Wörterbuch I - II. Heidelberg 1910, 1911.

FDGWb = Pekka Katara - Ingrid Schellbach-Kopra, Finnisch-deutsches Grosswörterbuch. Porvoo 1974.

Fritzner = Johan Fritzner, Ordbog over Det gamle norske Sprog II. Kristiania 1891.

FUF $=$ Finnisch-ugrische Forschungen. Helsinki.

Ganander $=$ Christfrid Ganander, Nytt Finskt Lexicon I - III. 1786-1787. Faksimile. Porvoo 1937 - 1940.

Grimm = Jacob Grimm und Wilhelm Grimm, Deutsches Wörterbuch V. Leipzig 1873.

Hakulinen 1960 = Lauri Hakulinen, Handbuch der finnischen Sprache II. Wiesbaden.

- 1979 = Lauri Hakulinen, Suomen kielen rakenne ja kehitys. Neljäs, korjattu ja lisätty painos. Keuruu.

Heggstad = Leiv Heggstad, Gammalnorsk ordbok med nynorsk tydning. Oslo 1963.

Helenius = Carl Helenius, Suomalainen ja Ruozalainen Sana-Kirja. Turku 1838.

Hellquist $=$ Elof Hellquist, Svensk etymologisk ordbok I - II. Andra upplagan. Lund 1939.

Hertzen 1973 = Erik v. Hertzen, Itämerensuomen lainasanakerrostumien ikäämisestä. JSFOu 72.

Hofstra $1980=$ Tette Hofstra, Ein germanisches Original für fi. pahna 'Stroh; Stall '. Finnisch-Ugrische Mitteilungen 4.

Holthausen $=$ F. Holthausen, Altenglisches etymologisches Wörterbuch. 2. Auflage. Heidelberg 1963.

IMS = Inkeroismurteiden sanakirja. Toimittanut R. E. Nirvi. Helsinki 1971.

Itkonen, T. 1962 = Terho Itkonen, Keidas. Erään maastotermin vaiheita. Suomi 110:1.

- $1972=$ Terho Itkonen, Historiantakaiset Häme ja Suomi kielentutkijan näkökulmasta. Historiallinen Aikakauskirja 2.

- 1973a $=$ Terho Itkonen, Über eine germanische Lehnwortsippe im Finnischen und Lappischen. FUF 40.

- 1973b = Terho Itkonen, Männyn selvittelyä. JSFOu 72.

- $1980=$ Terho Itkonen, Lisiä suomen rajausoppiin. Vir. 
Jóhannesson $=$ Alexander Jóhannesson, Isländisches etymologisches Wörterbuch. Bern 1956.

Jonsson 1966 = Hans Jonsson, Nordiska ord för vattensamling. Lundastudier i nordiska språkvetenskap 16. Lund.

JSFOu = Journal de la Société Finno-ougrienne. Helsinki.

Juslenius = Daniel Juslenius, Suomalaisen Sana-Lugun Coetus. Stockholm 1745. Näköispainos Henrik Gabriel Porthanille kuuluneesta välilehditetystä kappaleesta. Porvoo 1968.

Juslenius lis. = A. V. Koskimies, Lisäyksiä Jusleniuksen Sana-Lugun Coetukseen. Tehneet Salomon Kreander ja Juhana Canstrén. Suomi IV:16. Helsinki $1916-1917$.

Karsten $1943-1944=$ T. E. Karsten, Finnar och germaner. Folkmålsstudier IX - X. Helsingfors.

KKS = Karjalan kielen sanakirja I - II. Helsinki 1968, 1974.

KLM XXI = Kulturhistorisk lexikon for nordisk middelalder fra vikingetid til reformationstid XXI. København 1977.

Kluge - Mitzka $=$ Friedrich Kluge, Etymologisches Wörterbuch der deutschen Sprache. 20. Auflage. Bearbeitet von Walther Mitzka. Berlin 1967.

Kniezsa 1974 = István Kniezsa, A magyar nyelv szláv jövevényszavai $1-2$. Budapest.

Koivulehto 1971 = Jorma Koivulehto, 'Jäten' in deutschen Mundarten. Wortgeographisch-etymologische Untersuchungen. Annales Academiae Scientiarum Fennicae. Ser. B. Tom. 170. Helsinki.

- 1973 = Jorma Koivulehto, Germanisch-finnische Lehnbeziehungen III. Neuphilologische Mitteilungen 74.

- 1974 = Jorma Koivulehto, Lisiä germaanis-suomalaisiin lainakosketuksiin. Vir.

- 1978 = Jorma Koivulehto, "'Thomas L. Markey, Germanic Dialect Grouping and the Position of Ingræonic. Innsbruck 1976." Rezension in Neuphilologische Mitteilungen 80.

Krause 1971 = Wolfgang Krause, Die Sprache der urnordischen Runeninschriften. Heidelberg.

Kuusi 1953 = Vanhan kansan sananlaskuviisaus. Koonnut ja järjestänyt Matti Kuusi. Porvoo.

Laanest 1975 = Arvo Laanest, Sissejuhatus läänemeresoome keeltesse. Tallinn.

Lönnrot $=$ Elias Lönnrot, Suomalais-Ruotsalainen Sanakirja I - II. 1866 -1880. Faksimile. Lisävihko Elias Lönnrotin sanakirjaan. Toimittanut A. H. Kallio. Faksimile. Porvoo 1958.

LyMS = Lyydiläismurteiden sanakirja. Toimittanut ja julkaissut Juho Kujola. Helsinki 1944.

Mäkeläinen $1975=$ Osmo Mäkeläinen, Hulhavan jäljillä. Vir.

Matisto - Virkkala 1964 = Arvo Matisto - Kalevi Virkkala, Tampereen seudun geologia. Porvoo.

MNyTESz = A magyar nyelv történeti-etimológiai szótára II. Budapest 1970.

MSFOu = Mémoires de la Société Finno-ougrienne. Helsinki.

Nielsen $=$ Niels Åge Nielsen, Dansk etymologisk ordbog. 2. reviderede udgave med et tillæg. København 1969. 
Nirvi $=$ R. E. Nirvi, Kiihtelysvaaran murteen sanakirja II. Lappeenranta 1975.

Nissilä 1962 = Viljo Nissilä, Suomalaista paikannimitutkimusta. Helsinki.

- $1967=$ Viljo Nissilä, Virolaisia kosketuksia Suomen nimistössä. Kalevalaseuran vuosikirja 47.

$-1975=$ Viljo Nissilä, Suomen Karjalan nimistö. Vammala.

NS $=$ Nykysuomen sanakirja I - VI. Porvoo $1951-1961$.

NT = Nimitoimisto ("Namenbüro"). Forschungszentrum für die Landessprachen Finnlands. Helsinki.

Olsson 1959 = Ingemar Olsson, Gotländska terrängord. Acta Universitat is Stockholmiensis. Stockholm Studies in Scandinavian Philology. New Series 3.

ODS $=$ Ordbog over det danske Sprog, Udgivet af det Danske Sprog- og Litteraturselskab $1-27$. København $1919-1954$.

Pall 1969 = Valdek Pall, Põhja-Tartumaa kohanimed I. Tallinn.

Rapola 1956 = Martti Rapola, Antti Wikmanin sanakirja. Suomi 107:3.

Raun 1958 = Alo Raun, Über die ältesten germanischen Lehnwörter im Ostseefinnischen. Ural-Altaische Jahrbücher 30.

- 1971 = Alo Raun, Essays in Finno-Ugric and Finnic Linguistics. Indiana University Publications. Uralic and Altaic Series. Volume 107.

Raun - Saareste $1965=$ Alo Raun and Andrus Saareste, Introduction to Estonian Linguistics. Wiesbaden.

Renvall = Kustaa Renvall, Suomalainen Sana-Kirja. Turku 1826.

Saareste $=$ Andrus Saareste, Eesti keele mõisteline sõnaraamat I - II. Huddinge 1958, 1959.

- 1953 = Andrus Saareste, Länsi-Viron sanaston suhteesta suomen kieleen. Suomi 106:3.

Seebold $1970=$ Elmar Seebold, Vergleichendes und etymologisches Wörterbuch der germanischen starken Verben. The Hague-Paris.

Setälä $1913=$ E. N. Setälä, Bibliographisches verzeichnis der in der literatur behandelten älteren germanischen bestandteile in den ostseefinnischen sprachen. FUF 13.

SKES $=$ Y. H. Toivonen - Erkki Itkonen - Aulis J. Joki - Reino Peltola, Suomen kielen etymologinen sanakirja I - VI. Helsinki 1955 - 1975.

SMS = Suomen murteiden sanakirjan aineskokoelmat ("Wörterbucharchiv der finnischen Mundarten"). Forschungszentrum für die Landessprachen Finnlands. Helsinki.

Toivonen 1944 = Y. H. Toivonen, Etymologisia huomioita. Vir.

Torp = Alf Torp, Nynorsk etymologisk ordbok. Oslo 1919.

Vahtola 1980 = Jouko Vahtola, Tornionjoki- ja Kemijokilaakson asutuksen synty. Nimistötieteellinen ja historiallinen tutkimus. Studia historica septentrionalia 3. Kuusamo.

Wiedemann $=$ Ferdinand Wiedemann, Ehstnisch-deutsches Wörterbuch. Zweite vermehrte Auflage. St. Petersburg 1893.

Vilkuna, K. 1956 = Kustaa Vilkuna, Kiviraunion historia. Kotiseutu. Suomen Kotiseutuliiton aikakauslehti.

- 1968 = Kustaa Vilkuna, Vuotuinen ajantieto. Toinen, korjattu painos. Helsinki. 
Vir. = Virittäjä. Kotikielen Seuran aikakauslehti. Helsinki.

Vries 1962 = Jan de Vries, Altnordisches etymologisches Wörterbuch. Zweite verbesserte Auflage. Leiden.

- 1971 = Jan de Vries, Nederlands etymologisch woordenboek. Leiden.

Vuorela 1979 = Toivo Vuorela, Kansanperinteen sanakirja. Porvoo. 